\title{
Silent Growth of a "Super-Giant" Hemangioma
}

\author{
Daniela Falcão ${ }^{a}$ Isabel Pedroto ${ }^{a, b}$ Teresa Moreira ${ }^{a}$ \\ ${ }^{a}$ Gastroenterology Department, Centro Hospitalar e Universitário do Porto, Porto, Portugal; \\ ${ }^{\mathrm{b}}$ Instituto de Ciências Biomédicas Abel Salazar, Universidade do Porto, Porto, Portugal
}

\section{Keywords}

Hemangioma · Liver · Tumor · Growth · Pregnancy

\section{Crescimento silencioso de um Hemangioma "Super-Gigante"}

\section{Palavras Chave}

Hemangioma · Fígado · Tumor · Crescimento · Gravidez

A 29-year-old woman presented with a voluminous hepatic mass on a screening ultrasound performed during her second pregnancy. She underwent a magnetic resonance imaging scan that was consistent with a cavernous hemangioma measuring $250 \mathrm{~mm}$ in the largest diameter, with a nodular margin, hypointense images on T1-weighted sequences, hyperintense on T2-weighted sequences, and higher signal intensity on long repetition and echo times. This mass involved almost the entire right hepatic lobe with multiple infracentimetric hemangiomas in the left lobe. An analytical study showed discrete pancytopenia. Liver tests were within the normal range. Both her pregnancies were uneventful. Due to the absence of symptoms the patient was kept under clinical surveillance.

During 25 years of follow-up the patient remained asymptomatic, with a progressive increase of lesion dimen-

karger@karger.com www.karger.com/pjg

Karger

GOPEN ACCESS (c) 2021 Sociedade Portuguesa de Gastrenterologia Published by S. Karger AG, Basel

This is an Open Access article licensed under the Creative Commons Attribution-NonCommercial-4.0 International License (CC BY-NC) (http://www.karger.com/Services/OpenAccessLicense), applicable to the online version of the article only. Usage and distribution for commercial purposes requires written permission.

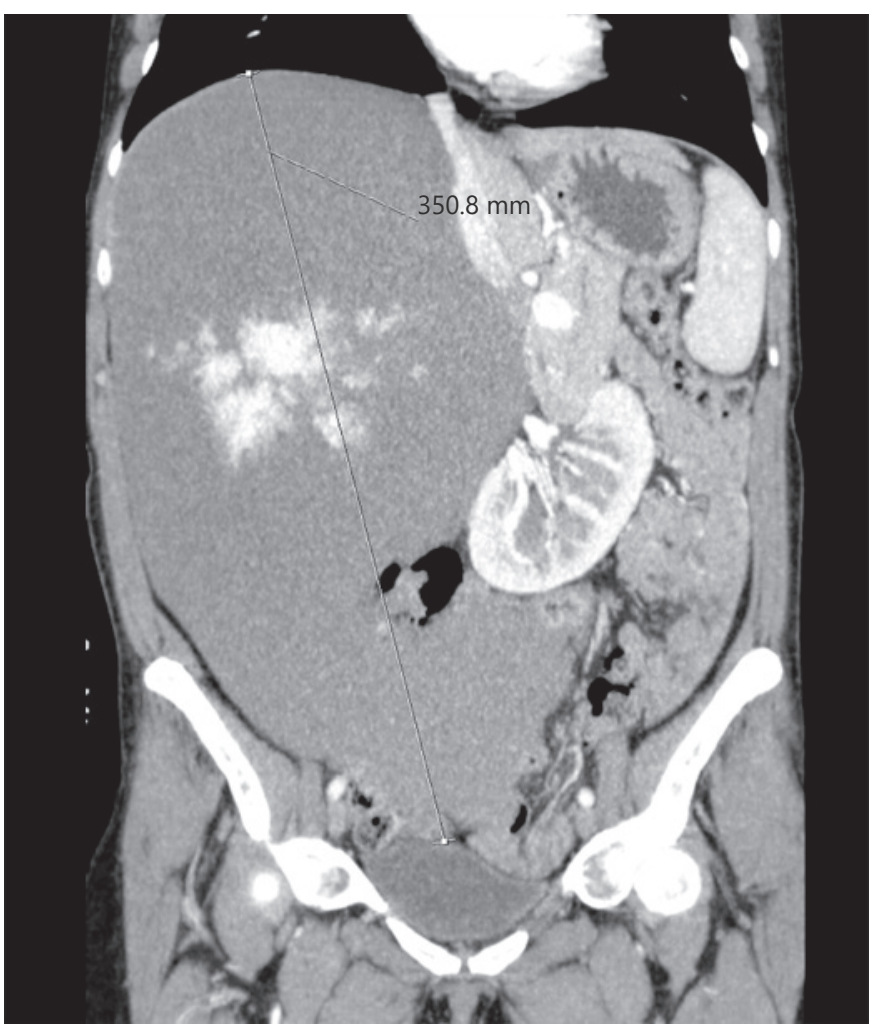

Fig. 1. Contrast-enhanced coronal computed tomography scan demonstrating the huge size of the hemangioma $(351 \times 180 \times$ $220 \mathrm{~mm}$ ), which occupies almost the entire right lobe. 
Fig. 2. Contrast-enhanced axial computed tomography scan showing the displacement of the pancreas, descending colon, small bowel, and the right kidney to the left side, due to the large size of the hemangioma in the right hepatic lobe. a Arterial phase. b Portal venous phase.
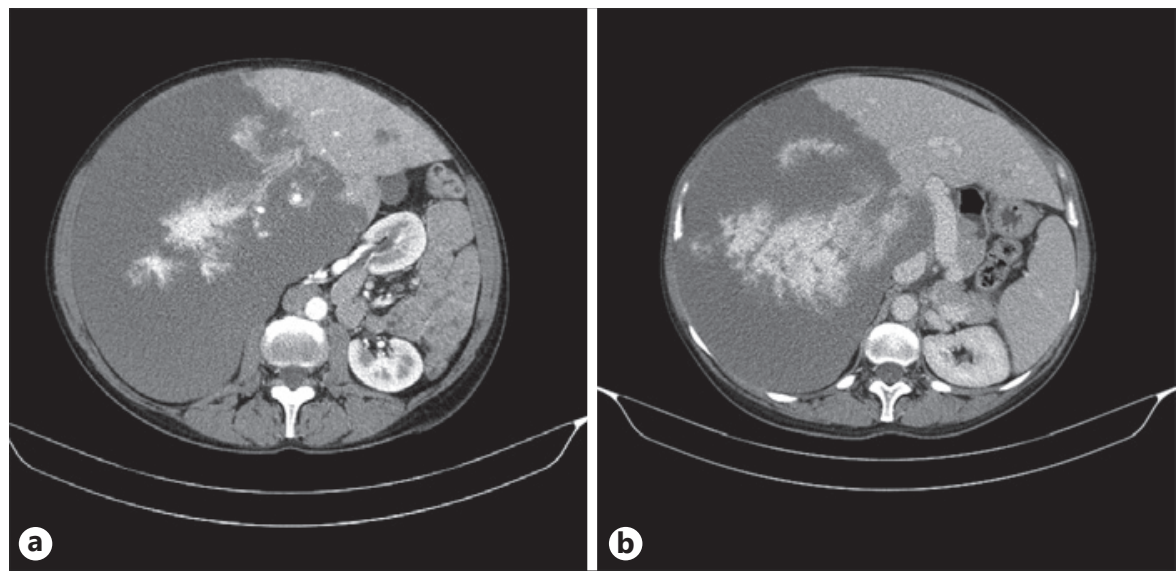

sions. A subsequent computed tomography scan revealed a hypodense mass with centrifugal enhancement during the late phase, an atypical presentation in relation to its dimensions $(351 \times 180 \times 220 \mathrm{~mm}$; Fig. 1$)$.

This massive liver lesion in the right lobe lead to considerable displacement of the pancreas, descending colon, small bowel, and the right kidney to the opposite side (Fig. 2). There were no signs of hepatic venous outflow obstruction, portal hypertension, or compression of the biliary tract.

When hemangiomas exceed $50 \mathrm{~mm}$, they are defined as giant lesions [1]. Here, we present a "super-giant" hemangioma, diagnosed during the second pregnancy of the patient, showing a slow growth rate and no complications after more than 2 decades of follow-up.

The influence of gender in liver hemangiomas appearance is undeniable according to a female:male ratio of up to $5: 1$ [2]. However, the role of female sex hormones and the effect of pregnancy on the evolution of hemangiomas is uncertain. There are reports of hemangioma complications during pregnancy such as rupture and intratumoral hemorrhage $[3,4]$, but growth of hepatic hemangiomas during pregnancy is difficult to estimate due to the lack of previous imaging evaluations. Giant hemangiomas should not preclude pregnancy, but close monitoring should be provided.

\section{Statement of Ethics}

The authors have no ethical conflicts to disclose. Informed consent was obtained from the patient for the publication of their information and imaging. Informed written consent was signed.

\section{Conflict of Interest Statement}

The authors declare that they do not have anything to disclose regarding funding or conflict of interest with respect to this manuscript.

\section{Funding Sources}

The authors have no funding sources to declare.

\section{Author Contributions}

The 3 authors contributed to the planning and organization of the article. D.F. was responsible for the first draft. I.P. and T.M. contributed to the improvement of the final draft and overall critical review.

References $\quad 1$ Ketchum WA, Lin-Hurtubise KM, Ochmanek E, Ishihara K, Rice RD. Management of symptomatic hepatic "mega" hemangioma. Hawaii J Med Public Health. 2019 Apr;78(4):128-31.

2 Reddy KR, Kligerman S, Levi J, Livingstone A, Molina E, Franceschi D, et al. Benign and solid tumors of the liver: relationship to sex, age, size of tumors, and outcome. Am Surg. 2001 Feb;67(2):173-8.

3 Sewell JH, Weiss K. Spontaneous rupture of hemangioma of the liver. A review of the literature and presentation of illustrative case. Arch Surg. 1961;83:729-33.

4 Graham E, Cohen AW, Soulen M, Faye R. Symptomatic liver hemangioma with intratumor hemorrhage treated by angiography and embolization during pregnancy. Obstet Gynecol. 1993 May;81(5):813-6. 